\title{
Mesenchymal stem cell therapies for skin repair and regeneration
}

\begin{abstract}
In the clinic, partial to full-thickness skin defects are generally treated through debridement and patching with autologous skin grafts. Autologous skin grafts are in severely limited supply, especially for ill patients lacking healthy tissue to donate to themselves. Stem cells have come into interest as a novel, viable way to heal skin wounds. Mesenchymal stem cells (MSCs) are of particular interest due to its common mesoderm germ layer origins as skin tissue. Mesenchymal stem cells are primarily derived from bone marrow, adipose tissue and umbilical cord. Bone marrow MSCs have been widely applied to chronic wounds in non-diabetic and diabetic subjects with positive results for wound healing and closure. Adipose-derived stem cells (ASCs) are a more recent application to skin healing. It has been found that ASCs secrete growth factors positive for augmenting wound healing, such as TGF- $\beta$. ASCs are also a more attractive cell source for clinical use due their great abundance and ease of procurement from discarded tissues from cosmetic surgery procedures. Most recently, umbilical cord MSCs have been tested for its effects on wound healing, showing that the cells paracrine effects can accelerate skin wound healing. Overall, mesenchymal stem cells show great promise to progress skin wound treatments, especially for chronic wounds. However, more clinical research is needed prior to patient application.
\end{abstract}

Keywords: skin, regeneration, repair, mesenchymal stem cell, bone marrow, adipose, umbilical cord
Volume I Issue 3 - 2017

\author{
Mai Lam T \\ Department of Biomedical Engineering, Wayne State University, \\ USA
}

Correspondence: Mai T Lam, Wayne State University, 818W. Hancock St., Detroit, MI, USA, Tel (3I3) 577-0I 18, (3।3) 5778333, Email mtlamdo@gmail.com

Received: June 20, 2017 | Published: August 29, 2017

\section{Introduction}

Skin damage from partial to full-thickness defects has limited treatment strategies in the clinic. Skin wounds are generally treated by debridement followed by patching with autologous skin grafts. However, supply of autologous skin grafts is limited, especially for larger wound coverage such as with burns. ${ }^{1}$ The field of tissue engineering has provided various biomaterial solutions to support wound healing..$^{2-7}$ Biomaterial approaches have included natural materials such as chitosan, collagen, degradable hydrogels, and protein glue; $;^{2-4}$ and synthetic materials such as polymer nanofibers, bioactive glass, and electrospun fibers. ${ }^{4-7}$ Biomaterials have had success in supporting wound healing as scaffolding materials for endogenous fibroblasts and keratinocytes as they infiltrate the wound and repair the skin. However, biomaterials are limited in their ability to stimulate the wound healing process wound repair.

Stem cell strategies have gained much popularity in recent years, encompassingnovel techniques to promote and accelerate wound healing. Mesenchymal stem cells (MSCs) are of particular interest, due to their availability and multipotent nature. In addition, mesenchymal stem cells originate from the mesoderm germ layer, sharing origins with skin. Stem cells from lineages similar to the tissue into which they are transplanted respond better to the analogous paracrine factors. Hence, mesenchymal stem cells are an ideal treatment strategy to repair and regenerate skin.

\section{Discussion}

\section{Bone marrow MSCs}

MSCs derive from two major sources: the bone marrow and adipose tissue. Bone marrow MSCs are isolated from bone marrow aspirates, involving a painful process where bone marrow is syringe extracted from the back of the pelvic bone. Only about $0.001 \%$ of bone marrow mononuclear cells in the aspirate are MSCs,${ }^{8}$ and efforts to increase cell number through cell culture expansion are complicated to apply to the clinic given the time needed and difficulty in maintaining stem cell phenotype in the lab. Clearly, supply of bone marrow is finite but is more readily available than pluripotent cells like embryonic stem cells. Bone marrow MSCs have been known to differentiate into a wide variety of mesenchymal cell types, including bone, cartilage and fat cells ${ }^{9-11}$ and even into cells beyond its originating phenotype, such as cardiomyocytes and neurons. ${ }^{12,13}$ In applications to the skin, bone marrow aspirate, bone marrow mononuclear cells, and a combination of cells and biomaterial have been used. ${ }^{14-17}$ In a study utilizing autologous bone marrow aspirate,,$^{14} 75$ patients with chronic wounds were enrolled. Inclusion criteria included wounds that did not respond to traditional treatment for at least 6 weeks, and sized $100 \mathrm{~cm}^{2}$ or less. Three study groups were formed based on treatment application to the wound: 1) fresh bone marrow aspirate, 2) cultured bone marrow, and 3 ) controls of saline. There was a significant change in wounds size at day 7 and week 4 . In a follow-up post week $4,60 \%$ of patients in group 1 and $76 \%$ of patients in group 2 did not need further wound care. In contrast, $80 \%$ of patients in the saline control group required surgical intervention. In a 16 month follow-up with group 2, the wound was completely healed with no signs of contracture.

Chronic wounds are a notorious complication for diabetic patients. Bone marrow MSCs have been explored as a treatment option. ${ }^{15-17}$ In one study, allogeneic MSCs were compared to the MSCs' acellular derivatives in a non-obese diabetic (NOD) mouse model..$^{15}$ The cells or acellular derivatives were intradermally injected around the wound site. Mice treated with the acellular derivative displayed significantly higher percentages of wound closure on days 4,6 , and 8 compared 
to wounds that received cells or control vehicles. Acellular products produced less pronounced inflammatory response, more granulation tissue, and a higher density of collagen fibers. This study suggests that growth factors present in bone marrow MSCs require time for cell release, whereas during the decellularization process these growth factors are released and readily available to stimulate the wound healing process.

\section{Adipose-derived MSCs}

While bone marrow MSCs can be difficult to obtain, adiposederived stem cells (ASCs) are much more abundant and are easily isolated from subcutaneous fat tissue and lipoaspirates. In addition, these adipose tissues are generally discarded from plastic surgery clinics, making excellent use of otherwise disposed tissue. ASCs have been widely explored for skin application given its common mesoderm layer origins. Biomaterial-delivered ASCs is a common approach, including stem cell delivery methods on extracellular matrix scaffolds, hydrogels, and biodegradable polymers. ${ }^{18-22}$ In one study, development of an injectable, thermo sensitive hydrogel was explored. ${ }^{22}$ A Pluronic F-127 hydrogel was used to encapsulate ASCs and applied topically to full-thickness cutaneous wounds in a mouse diabetic model. Mice given the ASC-hydrogel treatment experienced wounds with increased angiogenesis evidenced by the CD31 marker, enhanced cell proliferation evidenced by the Ki67 marker, and significantly increased wound closure rates. This study showed that ASCs can successfully be delivered to treat wounds via hydrogel.

Similar to the bone marrow acellular derivatives studied mentioned, exosomes derived from ASCs were investigated for their efficacy in skin wound treatment. Human ASCs were obtained and treated with an exosome precipitation solution. ${ }^{23}$ Full-thickness wounds were created on the backs of Balb/c mice and four groups treatment groups administered: 1) no treatment control, 2) saline injected control, 3) exosome in saline subcutaneous injection, and 4) exosome in saline intravenously injected. Bioluminesence imaging showed that exosomes injected systemically tracked to the wound site. Cutaneous wound healing was significantly accelerated in the exosome groups. Histology revealed increases in collagen I and II production in the early wound healing stages, whereas in the late wound healing stages to reduce scar formation the exosomes may inhibit collagen expression.

To create a 3D milieu, chitosan-hyaluronan spheroids containing ASCs were tested for effects on growth factor expression and hence enhanced wound healing capabilities. ${ }^{24}$ ASC spheroids were applied to a rat skin repair model with single cell ASCs serving as the control. Stem cells were administered to the wounds and covered with a hyaluronan gel/chitosan sponge to maintain moistness. Wounds treated with ASC spheroids exhibited accelerated wound healing and significantly increased angiogenesis. Nouveau microvessels coalesced around the spheroids. This study showed that a 3D environment can enhance the paracrine factor initiated by stem cells, resulting in augmented wound healing.

Though less commonly used, mesenchymal cells from the umbilical cord have found some application in skin wound healing. ${ }^{25-28}$ Human umbilical mesenchymal stem cells have been applied to wounds alone, or with extracellular matrix substrates, or in spheroid cultures. Umbilical MSCs treatment resulted in accelerated wound healing. Umbilical MSCs delivered in spheroids resulted in an increase in expression of several wound healing related growth factors, including
MMP-2, MMP-9, TGF- $\beta 1$, granulocyte-colony stimulating factor, FGF2, and IL-6.

\section{Conclusion}

Mesenchymal stem cells from bone marrow, adipose and umbilical cord sources are effective in accelerating wound healing. Paracrine factors expressed by these cells facilitate the stages of wound healing and have been found to allow for chronic wounds to heal. For mesenchymal stem cells to ubiquitously reach the clinic, methods to scale up supply of these stem cells and more cost-effective techniques for long term cell storage while maintaining stemness are needed. Further progression of this research to the manufacturing stages is needed in order to develop viable treatments applicable to the patient.

\section{Acknowledgements}

None.

\section{Conflict of interest}

The author declares no conflict of interest.

\section{References}

1. Sorkin M. Hair Follicle Specific ACVR1/ALK2 critically affects skin morphogenesis and attenuates wound healing. Wound Repair Regen. 2007;25(3):521-525.

2. Carvalho IC Mansur HS. Engineered 3D-scaffolds of photocrosslinked chitosan-gelatin hydrogel hybrids for chronic wound dressings and regeneration. Mater Sci Eng C Mater Biol Appl. 2017;78(1):690-705.

3. Jeon EY, Choi BH, Jung D, et al. Natural healing-inspired collagentargeting surgical protein glue for accelerated scarless skin regeneration. Biomaterials. 2017;134:154-165.

4. Zhou T, Sui B, Mo X, et al. Multifunctional and biomimetic fish collagen/ bioactive glass nanofibers: fabrication, antibacterial activity and inducing skin regeneration in vitro and in vivo. Int J Nano medicine. 2017;12:34953507.

5. Shoba E, Lakra R, Syamala Kiran M, et al. Fabrication of coreshell nanofibers for controlled delivery of bromelain and salvianolic acid B for skin regeneration in wound therapeutics. Biomed Mater. 2017;12(3):035005.

6. Yergoz F. Heparin mimetic peptide nanofiber gel promotes regeneration of full thickness burn injury. Biomaterials. 2017;134:117-127.

7. Mo Y. Controlled dual delivery of angiogenin and curcumin by electrospun nanofibers for skin regeneration. Tissue Eng Part A. 2017;23(13-14):597608 .

8. Madry H, Gao L, Eichler H, et al. Bone marrow aspirate concentrateenhanced marrow stimulation of chondral defects. Stem Cells Int. $2017 ; 1609685$

9. Aquino Martínez R, Artigas N, Gámez B, et al. Extracellular calcium promotes bone formation from bone marrow mesenchymal stem cells by amplifying the effects of BMP-2 on SMAD signalling. PLoS One. 2017;12(5): 0178158 .

10. Somoza RA. Transcriptome-wide analyses of human neonatal articular cartilage and human mesenchymal stem cells (hMSCs)-derived cartilage provide a new molecular target for evaluating engineered cartilage. Tissue Eng Part A. 2018;24(3-4):335-350..

11. Munir H. Adipogenic Differentiation of Mesenchymal Stem Cells Alters Their Immunomodulatory Properties in a Tissue-Specific Manner. Stem Cells. 2017;35(6):1636-1646. 
12. Shen X. Differentiation of mesenchymal stem cells into cardiomyocytes is regulated by miRNA-1-2 via WNT signaling pathway. J Biomed Sci. 2017;24(1):29

13. $\mathrm{Hu} \mathrm{Y}$. Effects of nerve growth factor and basic fibroblast growth factor dual gene modification on rat bone marrow mesenchymal stem cell differentiation into neuron-like cells in vitro. Mol Med Rep. 2016;13(1):4958 .

14. Gupta GJ, Karki K, Jain P, et al. Autologous bone marrow aspirate therapy for skin tissue engineering and tissue regeneration. Adv Wound Care (New Rochelle). 2017;6(4):135-142.

15. De Mayo T. The role of bone marrow mesenchymal stromal cell derivatives in skin wound healing in diabetic mice. PLoS One. 2017;12(6):e0177533.

16. Yan W. Raman spectroscopy enables noninvasive biochemical identification of the collagen regeneration in cutaneous wound healing of diabetic mice treated with MSCs. Lasers Med Sci. 2017;32(5):1131-1141.

17. Pouriran R. The effect of combined pulsed wave low-level laser therapy and human bone marrow mesenchymal stem cell-conditioned medium on open skin wound healing in diabetic rats. Photomed Laser Surg. 2016;34(8):345-354.

18. Matsumine H. Adipose-derived aldehyde dehydrogenase-expressing cells promote dermal regenerative potential with collagen-glycosaminoglycan scaffold. Wound Repair Regen. 2017;25(1):109-119.

19. Mineda K. Therapeutic potential of human adipose-derived stem/stromal cell microspheroids prepared by three-dimensional culture in non-crosslinked hyaluronic acid Gel. Stem Cells Transl Med. 2015;4(12):1511-1522.

20. Eke G, Mangir N, Hasirci N, et al. Development of a UV crosslinked biodegradable hydrogel containing adipose derived stem cells to promote vascularization for skin wounds and tissue engineering. Biomaterials. 2017;129:188-198.
21. Domingues JA. Bilaminar device of poly (lactic-co-glycolic acid)/collagen cultured with adipose-derived stem cells for dermal regeneration. Artif Organs. 2016;40(10):938-949.

22. Kaisang L. Adipose-derived stem cells seeded in Pluronic F-127 hydrogel promotes diabetic wound healing. J Surg Res. 2017;S0022 -S4804(17):30251-30252.

23. $\mathrm{Hu} \mathrm{L}$. Exosomes derived from human adipose mensenchymal stem cells accelerates cutaneous wound healing via optimizing the characteristics of fibroblasts. Sci Rep. 2016;6:32993.

24. Hsu SH, Hsieh PS. Self-assembled adult adipose-derived stem cell spheroids combined with biomaterials promote wound healing in a rat skin repair model. Wound Repair Regen. 2015;23(1):57-64.

25. Kusindarta DL. Human umbilical mesenchymal stem cells conditioned medium promote primary wound healing regeneration. Vet World. 2016;9(6):605-610.

26. Doi H. Potency of umbilical cord blood and Wharton's jelly-derived mesenchymal stem cells for scarless wound healing. Sci Rep. 2016;6:18844.

27. Nan W. Umbilical cord mesenchymal stem cells combined with a collagenfibrin double-layered membrane accelerates wound healing. Wounds. 2015;27(5):134-140.

28. Santos JM. Three-dimensional spheroid cell culture of umbilical cord tissue-derived mesenchymal stromal cells leads to enhanced paracrine induction of wound healing. Stem Cell Res Ther. 2015;6:90. 